Check for updates

Cite this: RSC Adv., 2017, 7, 52456

Received 9th August 2017

Accepted 6th November 2017

DOI: $10.1039 / c 7 r a 08796 a$

rsc.li/rsc-advances

\section{Surface decoration of selenium nanoparticles with curcumin induced HepG2 cell apoptosis through ROS mediated p53 and AKT signaling pathways $\uparrow$}

\author{
Min Guo, Yinghua Li, (D) Z Zhengfang Lin, Mingqi Zhao, Misi Xiao, Changbing Wang, \\ Tiantian Xu, Yu Xia and Bing Zhu (D) *
}

Hepatocellular carcinoma ( $\mathrm{HCC}$ ) is one of the most lethal cancers, and the morbidity and mortality are increasing continuously. Curcumin plays an important role in anticancer activity, while its clinical application is limited by its poor aqueous solubility. To develop an aqueous formulation and improve the anticancer activity of curcumin, curcumin-surface decorated selenium nanoparticles (Se@Cur) were designed in the present study. Transmission electron microscopy (TEM) images and MTT assays indicated SeaCur dramatically suppressed the proliferation of HepG2 cells and showed low toxicity to normal cells. Compared with SeNPs and curcumin, Se@Cur significantly inhibited the migration ability of HepG2 cells. Moreover, induction of apoptosis in HepG2 cells by Se@Cur was proved by accumulation of the sub-G1 cell population, nuclear condensation and activation of caspase-3. Furthermore, SeaCur promoted intracellular ROS overproduction and induced apoptosis via activating p53 and AKT signal pathways. Finally, in a xenograft nude mice model, Se@Cur suppressed the growth of tumors. Altogether, the findings in the present study demonstrated the application of Se@Cur as a safe and hopeful strategy for chemotherapeutics of HCC.

\section{Introduction}

Hepatocellular carcinoma (HCC) is considered as one of the most common malignancies and ranks third in cancerassociated deaths around the world. ${ }^{1-4}$ About 250000 new cases of HCC increase every year, with nearly 6000000 deaths occurring. ${ }^{5}$ The conventional therapies at the early stages of HCC include surgical restriction, interventional therapy, radiotherapy and radiofrequency ablation. ${ }^{6,7}$ The survival rate improves significantly after surgical restriction at the early phase of HCC. ${ }^{8,9}$ Unfortunately, the majority of patients with HCC were advanced stage at the time of diagnosis without proper screening tests and effective surveillance strategies. ${ }^{10}$ Besides, due to its insensitivity to chemotherapy and high metastatic potential, the survival of patients with HCC is very poor. Moreover, chemotherapy drugs possess potential drawbacks, such as short half-life, poor aqueous solubility and significant toxicity. Thus, it is urgent to develop novel therapeutic agents for the treatment of HCC. ${ }^{11}$

Curcumin, a hydrophobic polyphenol derived from turmeric Curcuma longa, has wide biological activities, such as

Center Laboratory, Guangzhou Women and Children's Medical Center, Guangzhou Medical University, Guangzhou, 510120, China. E-mail: zhubing2016@hotmail.com † Electronic supplementary information (ESI) available. See DOI: 10.1039/c7ra08796a

\$ Min Guo and Yinghua Li contributed equally to this work. anticancer, antioxidant, antibacterial, antifungal and antiviral. ${ }^{12-15}$ Curcumin plays a role in anticancer activity by regulating the expression of several cellular targets like AKT, MAPK, p53, AMPK and NF-кB, and thus controls cancer cell proliferation, growth, invasion and induces apoptosis. ${ }^{16,17}$ Furthermore, owing to no signs of toxicity in humans or animals treated with curcumin, it was proved to be a natural and safe agent. ${ }^{18}$ Unfortunately, the main problems for the clinical application of curcumin are compromised by its low oral bioavailability, rapid degradation and poor solubility in aqueous solution. ${ }^{19-21}$ Therefore, an aqueous formulation of curcumin is required for clinical application.

Nanotechnology has already been implied in different medical aspects, with a focus on the diagnosis of diseases and drug delivery systems for therapy of diseases. ${ }^{22-24}$ As potential drug delivery systems, nanomaterial has the characteristics of smart, surface functionalization and well soluble in aqueous solution, which provides a crucial way to develop aqueous formulations of hydrophobic drugs. ${ }^{25}$ Selenium is an important dietary trace element and can inhibit the growth of cancer cells. $^{26-28}$ Numerous reports have shown that selenium is a potent cancer chemopreventive agent and is associated with risk of many cancers, such as stomach, bladder, prostate, ovarian, colon, lung, breast cancer, and so on..$^{29-32}$ Due to the unique properties of selenium nanomaterial, it is applied in medicine, especially cancer gene and drug delivery. ${ }^{33}$ Thus, curcumin-loaded SeNPs nanoparticle (Se@Cur) was designed 
and synthesized as a potential cancer therapy against hepatocellular carcinoma.

The reactive oxygen species (ROS) play an important role in many physiological processes. The redox imbalance is associated with much pathology, such as cancer and other diseases. ${ }^{34}$ ROS are generated by the chemical reduction of $\mathrm{O}_{2}$, including superoxide $\left(\mathrm{O}_{2}{ }^{\circ}\right)$, superoxide anion $\left(\mathrm{O}_{2}{ }^{-}\right)$, hydroxyl $\left({ }^{\circ} \mathrm{OH}\right)$ and hydrogen peroxide $\left(\mathrm{H}_{2} \mathrm{O}_{2}\right)$. ROS plays a role of cell-signaling proteins, which are involved in growth, differentiation, and apoptosis of cell. ${ }^{35,36}$ Recent studies have indicated that ROS have impacts on many signaling pathways, such as MAPKs, NF$\kappa \mathrm{B}$, Keap1-Nrf2-ARE, PI3K-AKT, etc. ${ }^{37}$ In this study, Se@Cur was designed as a new chemotherapeutic agent to achieve HepG2 cells apoptosis and its underlying anticancer mechanisms through ROS-mediated signaling pathway were also explored.

\section{Experimental}

\subsection{Materials}

HepG2 cells and LO2 cells were acquired from American Type Culture Collection (ATCC, Manassas, VA). Fetal bovine serum (FBS) and Dulbecco's modified Eagle's medium (DMEM) were obtained from Gibco. P53, AKT and cleaved caspase-3 antibody were provided by Cell Signaling Technology Inc. (Danvers, MA, USA). $\mathrm{Na}_{2} \mathrm{SeO} 3$, Vitamin $\mathrm{C}$ (VC), coumarin-6, thiazolyl blue tetrazolium bromide (MTT) were acquired from Sigma-Aldrich (EMD Millipore, Billerica, MA, USA). BCA protein assay kit, Reactive Oxygen Species Assay Kit and caspase-3 activity assay kit were purchased from Beyotime Institute of Biotechnology (Shanghai, People's Republic of China). Mice were obtained from the Guangdong Medical Laboratory Animal Center (Guangdong, China). All animal procedures were performed according to the guidelines of Guangdong Medical Laboratory Animal Center.

\subsection{Synthesis and characterization of Se@Cur}

After SeNPs were synthetized as previous. ${ }^{38} 1 \mathrm{mM}$ curcumin was added into the SeNPs under magnetic stirring for half an hour. Then the Se@Cur complex was purified and re-dispersion in Milli-Q water. Finally, the production was dialyzed and measured by ICP-MS analysis. The particle morphology, elemental composition analysis, size distribution, zeta potential and stability in aqueous solutions of Se@Cur were analyzed by transmission electron microscopy (TEM), Energy Dispersive Xray (EDX) and Malvern Zetasizer Nano ZS particle analyzer, respectively. FT-IR samples were recorded using the KBr-disk method on Equinox 55 IR spectrometer. XPS measurement was carried out on an ESCALab 250 spectrometer.

\subsection{Cell culture and MTT assay}

The HepG2 and LO2 cells were maintained in DMEM supplemented with $10 \%$ FBS, $100 \mathrm{U} \mathrm{ml}^{-1}$ penicillin and $50 \mathrm{U} \mathrm{ml}^{-1}$ streptomycin at $37{ }^{\circ} \mathrm{C}$ in a humidified incubator (Thermo Scientific, USA) with $5 \% \mathrm{CO}_{2}$. The cytotoxicity and viability were evaluated by MTT assay. ${ }^{39}$ HepG2 and LO2 cells were seeded in triplicate in 96 well flat bottom respectively. Thereafter, the cells were exposed to SeNPs, curcumin, Se@Cur at different concentrations for $48 \mathrm{~h}$. After treatment, cells were incubated with MTT regent for $5 \mathrm{~h}$. Then the culture medium was removed and $150 \mu \mathrm{l}$ DMSO were added to each well. The conversion of MTT was read at $570 \mathrm{~nm}$ absorbance by microplate reader.

\subsection{Scratch assay}

The anti-cell migration effect of Se@Cur on HepG2 cells was detected by a scratch assay. In brief, after the cell confluence reached $80 \%$, cell monolayers were wounded with a sterile microtip and washed with PBS to discard detached cells. Then the cells were treated with Se, curcumin, Se@Cur and incubated for $24 \mathrm{~h}$. After that, the wound closure was observed and photographed by an Olympus microscope at 0 and $24 \mathrm{~h}$.

\subsection{Cell cycle assay}

The cell cycle assay was performed as describe previously. ${ }^{40}$ After treatment with SeNPs, curcumin and Se@Cur for $48 \mathrm{~h}$, the HepG2 cells were harvested by trypsinization. Then, the collected cells were resuspended in ice cold $70 \%$ ethanol and stored over night at $-20^{\circ} \mathrm{C}$ for fixation. Then the fixed cells were incubated with Propidium iodide at room temperature for 30 min away from light. The cells were measured by flow cytometry. Cell cycle phase quantification was analyzed by using ModFit LT 3.1 software.

\subsection{Measurement of reactive oxygen species (ROS) level}

The level of intracellular ROS which is induced by Se@Cur is detected as describe previously. ${ }^{41,42}$ In brief, after treatment with Se@Cur in different time, the HepG2 cells were stained with DCFH-DA $(10 \mu \mathrm{M})$ for $30 \mathrm{~min}$ in the dark. The ROS was detected by a fluorescence microscope. The same exposition time was acquired for all treatment groups to capture images. The fluorescence intensity was determined by a fluorescence plate reader with an excitation wavelength of $488 \mathrm{~nm}$ and emission wavelength of $525 \mathrm{~nm}$ respectively.

\subsection{Activation of caspase-3}

Caspase-3 activity was measured according to the methods described in our previous paper. ${ }^{43,44}$ Cell lysates, detection buffer and specific caspase substrates Ac-DEVD-pNA ( $2 \mathrm{mM})$ were added into a 96-well plate for $2 \mathrm{~h}$ at $37^{\circ} \mathrm{C}$. The activation of caspase- 3 was measured through the conversion of pNA, which was measured by $405 \mathrm{~nm}$ absorbance using a microplate reader.

\subsection{Western blotting analysis}

The expression of proteins associated with different signaling pathways after treatment with Se@Cur in HepG2 cells were analyzed by Western blot as previously reported..$^{45,46}$ In brief, HepG2 cells were lysed with RIPA solution after treatment with Se@Cur for $48 \mathrm{~h}$, followed by concentration detection of the proteins with a BCA kit. After that, equivalent amount of proteins were loaded in SDS-PAGE, then transferred to polyvinylidene difluoride membranes, and finally visualized by $\mathrm{X}$ ray film. 


\subsection{HepG2 cell xenograft mice model}

32 female nude mice were subcutaneously injected into the right armpit region with harvested HepG2 cells. When the volume of tumors reached about $75 \mathrm{~mm}^{3}$, the tumor-bearing mice were acquired. After that, the tumor-bearing mice were randomly allocated into a control group and 3 treatment groups. For study, tumor-bearing mice were treated with Se@Cur at dosages of $3 \mathrm{mg} \mathrm{kg}{ }^{-1}, 6 \mathrm{mg} \mathrm{kg}^{-1}$ and $9 \mathrm{mg} \mathrm{kg}^{-1}$ through intravenous injection every 2 days and continued for 16 days. As the control group, the mice were treated with equal volume of PBS $(0.2 \mathrm{ml}) .16$ days later, the tumor-bearing mice were killed, of which the tumor weight and body weight were measured. The animal experiments were performed following an approved protocol of Animal Experimentation Ethics Committee.

\subsection{Statistical analysis}

All results were all obtained from three independent tests and the data was analyzed using SPSS (ver. 19.0). Continuous variables were presented as mean \pm SD. The differences among multiple groups were compared using one-way analysis of variance (ANOVA). The probability of $P$ value of $<0.05\left(^{*}\right)$ and $P$ value of $<0.01(* *)$ were deemed to be significant in all experiments.

\section{Results and discussion}

\subsection{Preparation and characterization of Se@Cur}

In present study, we constructed a chemical method to synthesize selenium nanoparticle coated with curcumin (Se@Cur) and the synthetic route was shown in Fig. 1. The morphology of SeNPs and Se@Cur was characterized by TEM firstly. As shown in Fig. 2A and B, SeNPs and Se@Cur all presented small and sphere particles. While, compared with SeNPs, Se@Cur was more uniform and dispersive. As shown in Fig. 2C, the EDX analysis of Se@Cur showed the presence of three main signals, including Se atoms signal from SeNPs (35.8\%), O (38.2\%) and C (26\%) that from curcumin. Fig. 2D and $\mathrm{E}$ presented the size graphs of SeNPs and Se@Cur. SeNPs without curcumin decorated showed small sphere particles with a size of $141 \mathrm{~nm}$, while Se@Cur apparently reduced the size to $53 \mathrm{~nm}$ (Fig. 2D and E). Se@Cur with the property of small size enhanced the cellular uptake and was more easily entered into cell membrane. Besides, the absolute value of zeta potentials generated by SeNPs was $24.3 \mathrm{mv}$, and increased to $36.9 \mathrm{mV}$ when capped with curcumin (Fig. 2F). The results indicated that after surface modification with curcumin, Se@Cur showed higher stability than SeNPs. Furthermore, from Fig. 2G, the size of Se@Cur gradually increased and kept stable for at least 30 days. Taken together, the characterization of Se@Cur with small size and low zeta potential effectively promoted cellular uptake and nanostructures stability.

\subsection{The structure of Se@Cur}

The FTIR spectrum of Se@Cur gave clear evidence that curcumin ligand formed part of the nanocomposite. Curcumin

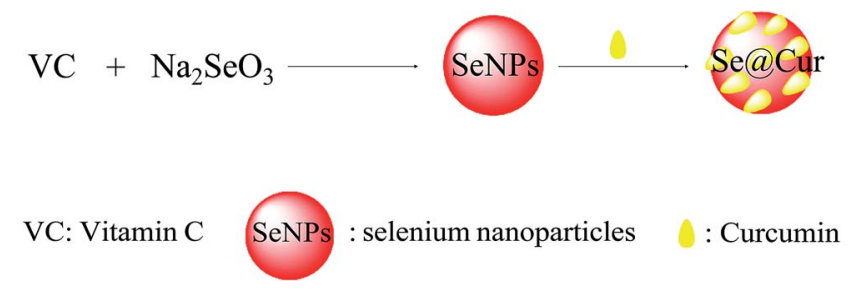

Fig. 1 Synthetic route of SeaCur.

displays IR absorbance peaks at $1269 \mathrm{~cm}^{-1}$ and $753 \mathrm{~cm}^{-1}$ corresponded to $-\mathrm{OH}$ and $-\mathrm{C}-\mathrm{H}$. The absence of these peaks in Se@Cur indicated the formation of Se@Cur (Fig. 3A). The XPS spectra were also recorded to examine the interaction between SeNPs and curcumin. As shown in Fig. 3B, the O 1s peak in the spectrum of Se@Cur further confirmed that curcumin had been successfully conjugated to the SeNPs, the results support the formation of Se-O bond in Se@Cur.

\subsection{In vitro cytotoxicity and anti-proliferation activity of Se@Cur}

To measure the viability and anti-proliferation activity of HepG2 cells after treatment with SeNPs, curcumin and Se@Cur, the MTT assay was performed. As shown in Fig. 4A, the cell survival rate of LO2 cells was higher than HepG2 cells when exposed to SeNPs $(88.45 \%$ vs. $86.94 \%)$, curcumin (80.65\% vs. $66.72 \%)$ and Se@Cur (74.95\% vs. $52.29 \%)$. Compared with SeNPs and curcumin, Se@Cur remarkably suppressed the viability of HepG2 cells and showed less cytotoxicity to normal cells. As shown in Fig. 4B, after treatment with SeNPs, curcumin and Se@Cur, the decrease of cell number, cell rounding, cell vacuoles and cytoplasm shrinkage were observed in different degree. The effects of SeNPs, curcumin and Se@Cur on the growth of HepG2 cells were also further confirmed. Synergy was evaluated by calculation of in vitro fractional inhibitory concentration-index values: minimum inhibitory concentration (MIC) of drug A combination present in Se@Cur of SeNPs $(125 \mu \mathrm{M})$; MIC of drug B combination present in Se@Cur of Cur $(20 \mu \mathrm{M})$; MIC of drug A alone corresponded to free SeNPs (1 mM); MIC of drug B alone corresponded to free Cur $(62.5 \mu \mathrm{M})$. Fractional IC (FIC) was calculated as (MIC drug A combination/MIC drug A alone) + (MIC drug B combination/MIC drug B alone) $=125 \mu \mathrm{M} / 1 \mathrm{mM}$ $+20 \mu \mathrm{M} / 62.5 \mu \mathrm{M}=0.445$. FIC was 0.445 , below 0.5 , indicating synergy. In this study, the FIC index was basically interpreted as follows: FIC < 0.5, synergy; FIC 0.5-2, indifference; FIC $>2$, antagonism. These results indicated Se@Cur suppressed the proliferation of HepG2 cells most effectively and induced more cancer cells death than non-cancer cell.

\subsection{Se@Cur inhibited the migration of HepG2 cells}

As present in Fig. 5, the HepG2 cells were exposed to SeNPs, curcumin, Se@Cur and the cellular migration was analyzed by a scratch assay. In the control group, the migrating cell was almost completely occupied the wounded gap after $24 \mathrm{~h}$. While, 
A
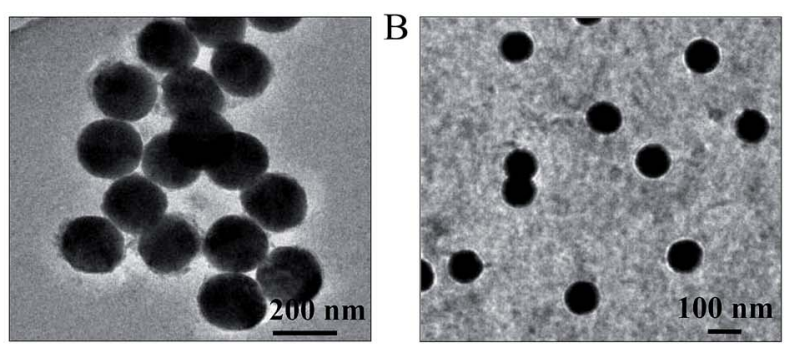

C

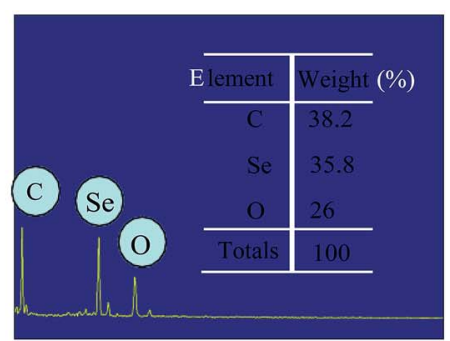

D

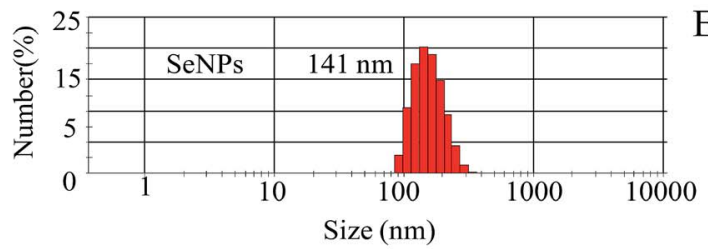

F

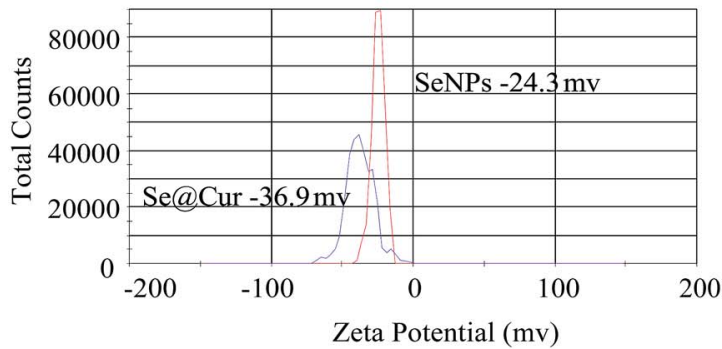

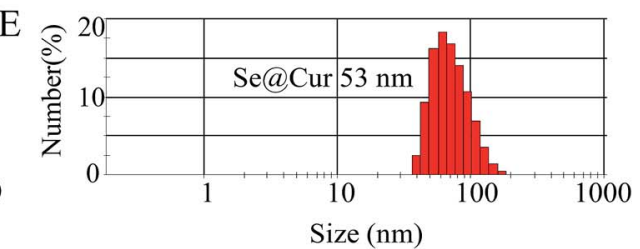

G

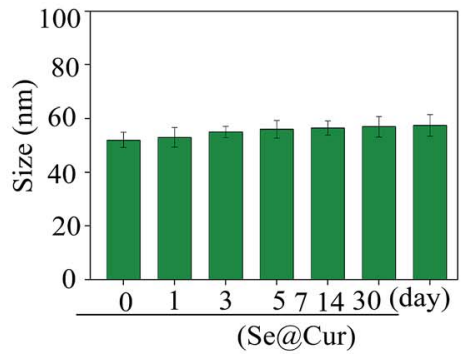

Fig. 2 Characterization of SeNPs and Se(Cur. (A) Representative TEM images of SeNPs and (B) SeaCur. (C) Representative EDX analysis of SeaCur. (D) Size distribution of SeNPs and (E) Se@Cur. (F) Zeta potentials of SeNPs and Se(aCur. (G) Stability of Se@Cur in aqueous solutions.

in the Se@Cur treated group, this gap was not occupied by migrating cells. The Migratory ratio of control, SeNPs, curcumin and Se@Cur in Fig. S1.† This result suggested that Se@Cur significantly inhibited the migration ability of HepG2 cells.

\subsection{Se@Cur inhibited HepG2 cells proliferation by induction of apoptosis}

Most anticancer drugs can induce cancer cells apoptosis which is an important mechanism for anticancer effect. In order to prove whether apoptosis was involved in HepG2 cell death induced by SeNPs, curcumin, and Se@Cur, a cell cycle assay was analyzed by flow cytometry. As shown in Fig. 6, the results revealed that the sub-G1 apoptotic cell population was significantly increased in the DNA histogram in different treatments. For instance, the exposure of HepG2 cells to different treatments of control, SeNPs, curcumin, and Se@Cur significantly increased in apoptotic population from 1.83 to $20.63 \%$. Compare to control group, Se@Cur treatment group had a significantly higher percentage of cells in the
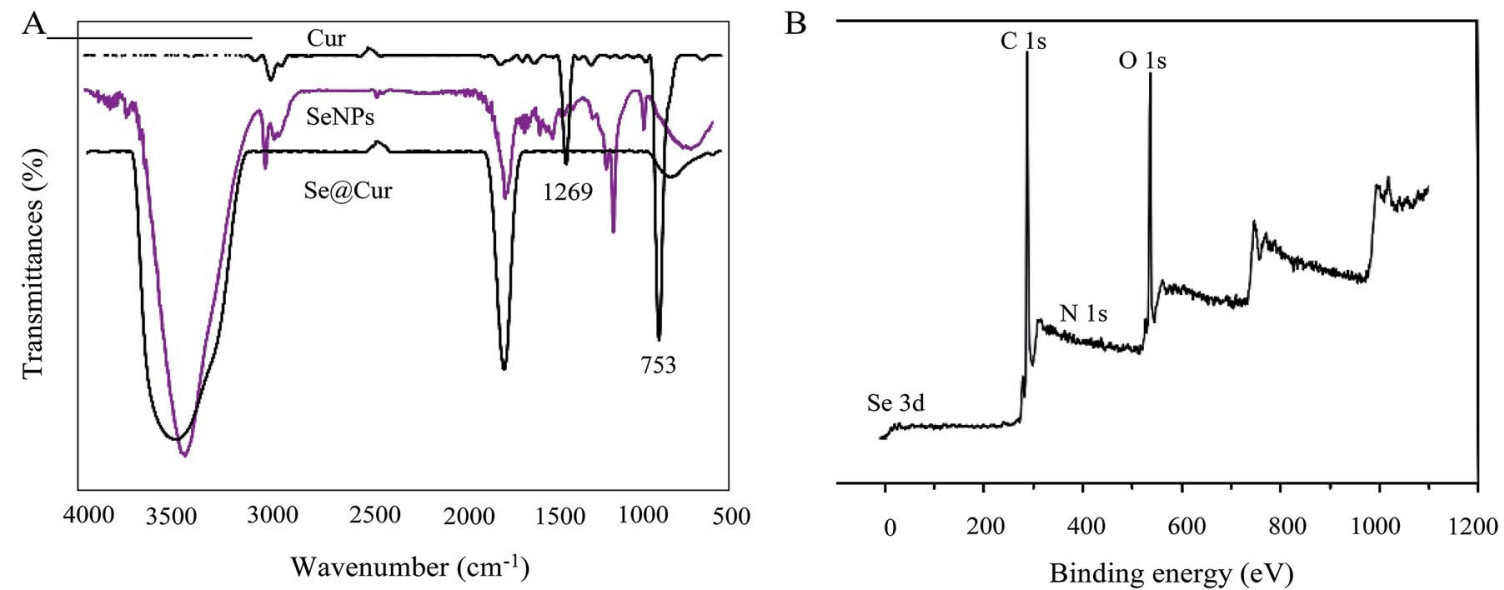

Fig. 3 FTIR spectrum and XPS detection of SeNPs and Se@ZNV. (A) FTIR spectrums. (B) XPS detection. 
A

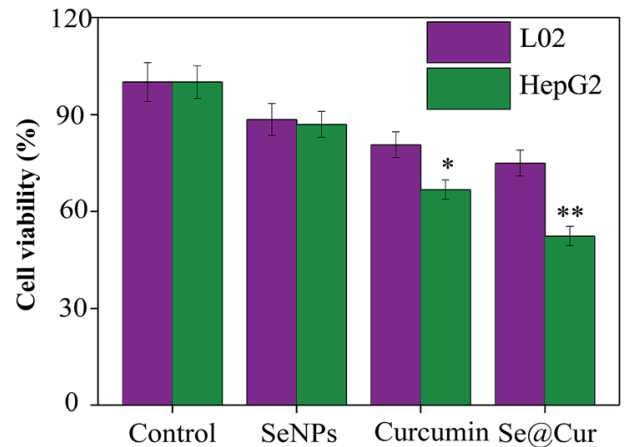

B Control

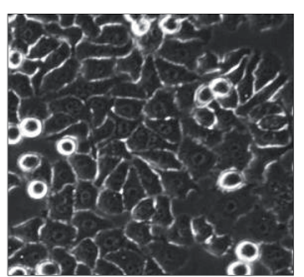

SeNPs

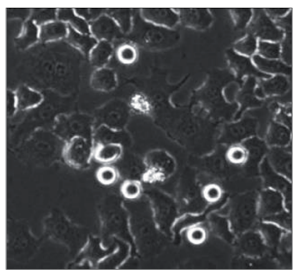

Curcumin

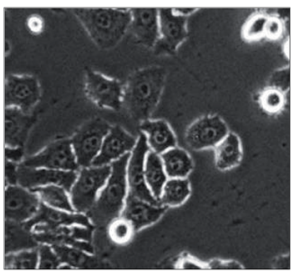

Se $@$ Cur

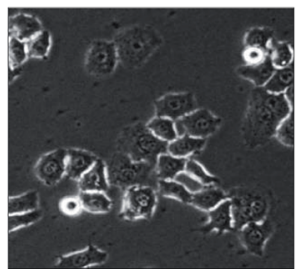

Fig. 4 Cytotoxic effects of Se@Cur on HepG2 cells. (A) Cell viability of Se(C Cur treated HepG2 cells and normal cells were determined by MTT assay. (B) After treatment with SeaCur, the morphological changes of HepG2 cells included cell number reduction with cell rounding, cell vacuoles and cytoplasm shrinkage.
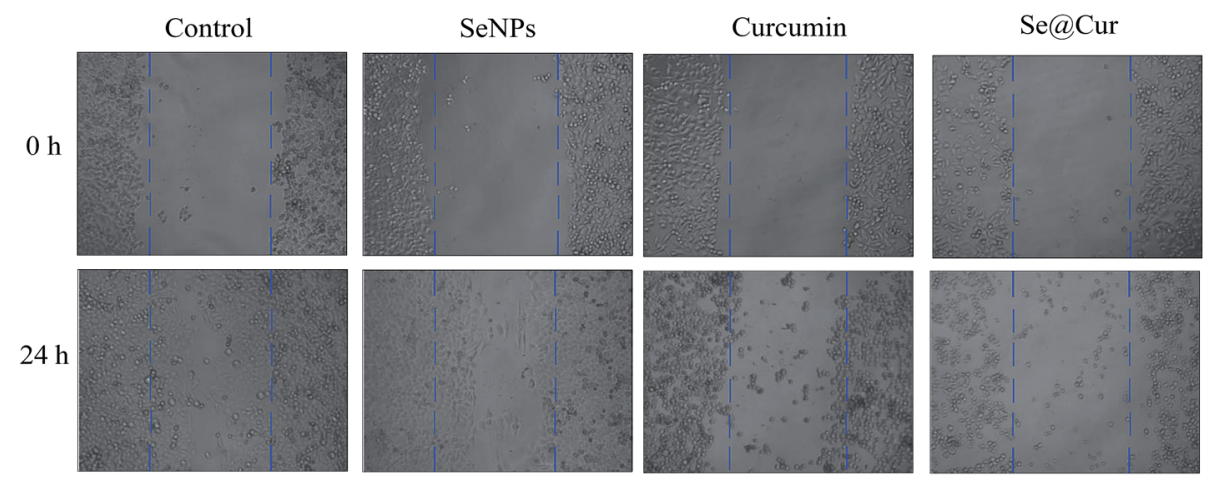

Fig. 5 A wound-healing assay was conducted to analyze cell migration. The migration of HepG2 cells was dramatically suppressed after treatment with SeaCur for $24 \mathrm{~h}$.

$S$ phase indicating inhibition the proliferation of HepG2 cells. Translocation of phosphatidylserine induced by SeNPs, curcumin and Se@Cur in HepG2 cell was showed in Fig. S2. $\dagger$ Those results suggested that Se@Cur induced HepG2 cells apoptosis and S phase cell cycle arrest.
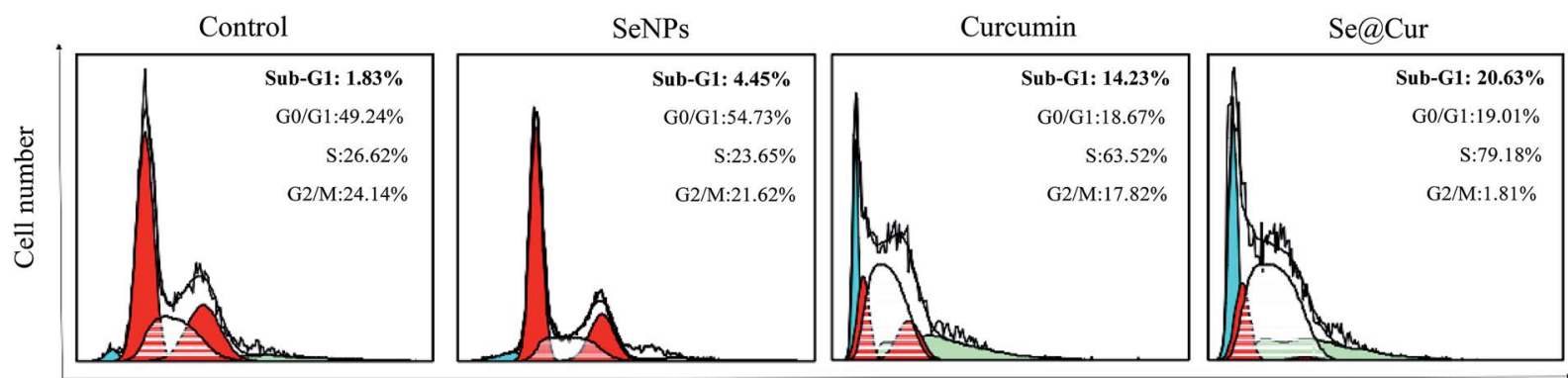

NDA content

Fig. 6 Effect of SeaCur on cell cycle progression in HepG2 cells measured by flow cytometry analysis. DNA quantitative analysis of cell cycle distribution in HepG2 cells treated with SeNPs, curcumin, and Se@Cur for $48 \mathrm{~h}$. SeNPs alone is $1 \mathrm{mM}$ curcumin alone is $62.5 \mu \mathrm{M}$ SeNPs combination present in SeaCur is $0.125 \mu \mathrm{M}$ curcumin combination present in Se@Cur is $20 \mu \mathrm{M}$. 
A

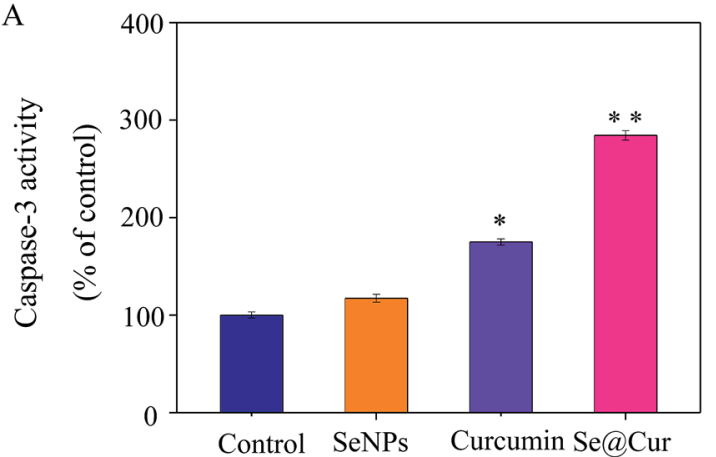

B

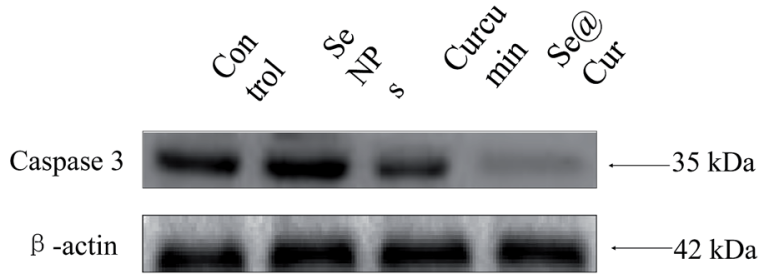

Fig. 7 SeaCur induced caspase activation. (A) HepG2 cells were treated with different treatments for $24 \mathrm{~h}$ and activation of caspase 3 was measured. (B) The expression level of caspase 3 protein was measured by western blotting.

\subsection{Activation of caspase-3 by Se@Cur}

Caspases are some family of cysteine proteases and important components in response to apoptosis. Caspase-3 has been regarded as the main executioner caspases in the cell, which lead to cell disassembly and DNA fragmentation. As an effector caspase, activation of caspase-3 was engaged by activation of initiator caspases through extrinsic or intrinsic apoptosis pathways. Caspase-3 involved in proteolytic cleavage of some important proteins. Activation of caspase-3 was measured by western blotting and fluorometric test. Compared to control group, the activation of caspase- 3 was significantly increased to $117 \%, 175 \%$, 285\% when the HepG2 cells were exposed to SeNPs, curcumin, Se@Cur, respectively (Fig. 7A). As shown in Fig. 7B, the level of caspase-3 expression was downregulated with treatment of Se@Cur. The results above suggested that nanosystem markedly increased the activation of caspase- 3 to mediate apoptosis.

\subsection{Induction of ROS in HepG2 cells by Se@Cur}

Caspase-3 was confirmed as an important mediator of cell apoptosis.

It has been reported that ROS was a vital regulator of cell signaling pathways triggered by anticancer drugs. In order to investigate weather Se@Cur could trigger ROS-mediated apoptosis, the ROS level was detected by DCF fluorescence assay. After exposure to SeNPs, curcumin, and Se@Cur, the ROS generation of HepG2 cells markedly elevated to $120 \%, 160 \%$ and $220 \%$ of the control group, respectively (Fig. 8A). From photos in Fig. 8B, the average fluorescent intensity of HepG2 cells treated with Se@Cur was the strongest, followed by curcumin and SeNPs treatment group. TEM image of HepG2 cells treated with Se@Cur was showed in Fig. S3. $\dagger$ These results revealed that the involvement of ROS in the anticancer action of Se@Cur.

\subsection{ROS-mediated signaling pathways by Se@Cur}

Furthermore, the molecular mechanisms of Se@Cur in inducing HepG2 cells apoptosis were investigated. Intracellular ROS overproduction could cause DNA damage response and lead to cell apoptosis via regulation of multiple signaling pathways, including JNK, AKT, ERK and P53, et al. ${ }^{47,48}$ The
A

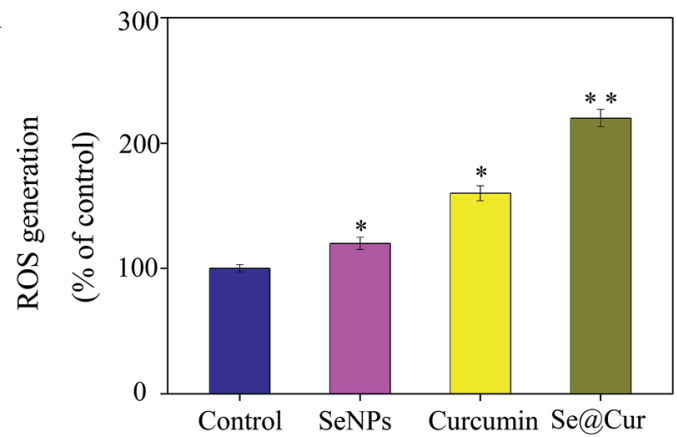

B

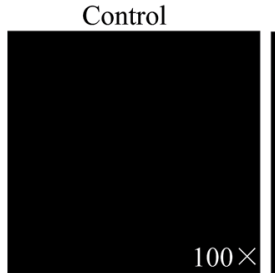

SeNPs

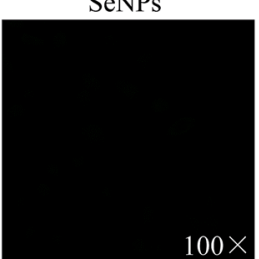

Curcumin

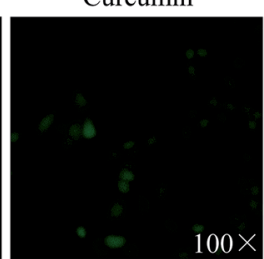

Se@Cur

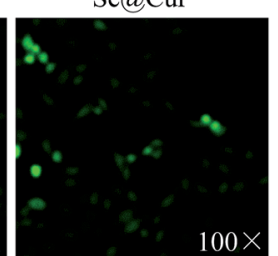

Fig. 8 The intracellular ROS of HepG2 cells exposed with Se@Cur. (A) ROS overproduction induced by Se@Cur was measured by a fluorescence plate reader. (B) Representative fluorescence images of cells stained by DCFH-DA. 
A

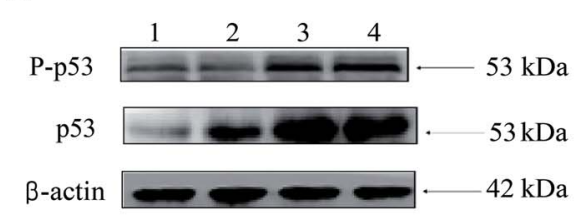

B

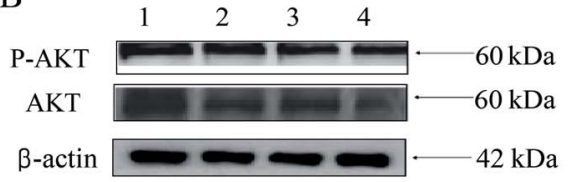

$\mathrm{C}$

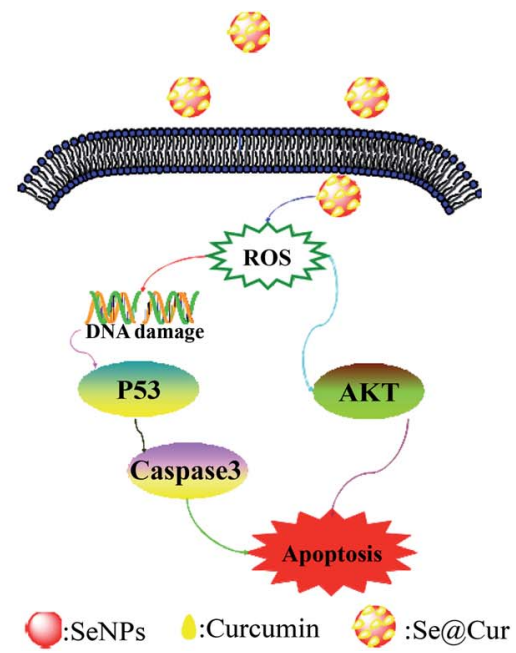

(1:Control 2:SeNPs 3:Cur 4.Se@Cur)

Fig. 9 Activation of ROS-mediated apoptosis signal pathways by Se(C) in HepG2 cells. (A) Activation of p53 signaling pathway. (B) Activation of AKT signal pathway. (C) The main signal pathway of ROS-mediated p53, AKT pathways.
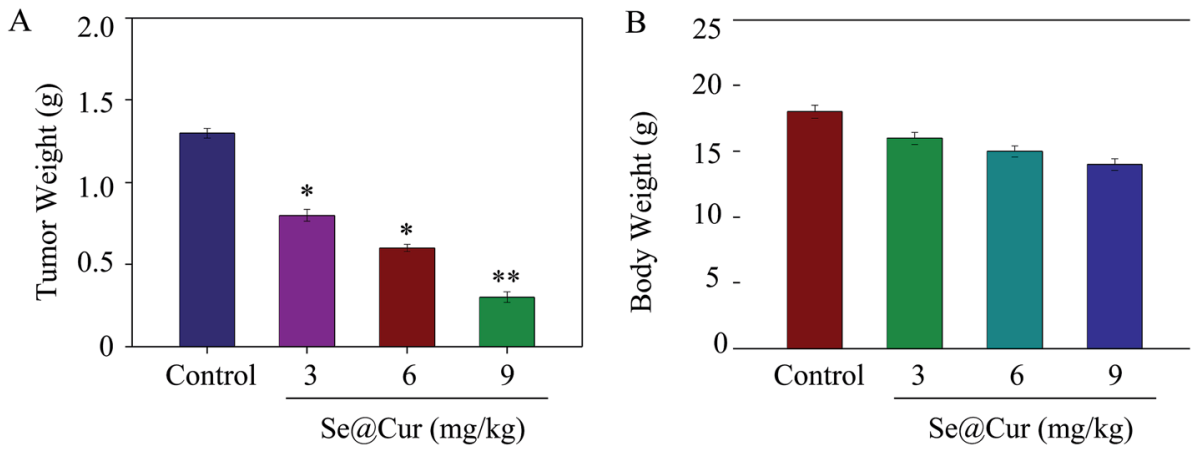

Fig. 10 Effects of anticancer of SeaCur in vivo. (A) Tumor weight of xenogragt HepG2 tumor nude mice after treatment with different concentration of SeaCur. (B) Body weight of xenograft HepG2 tumor nude mice after treatment with different concentration of SeaCur.

overproduction of ROS were significantly generated in cells exposed to Se@Cur as aforesaid, so the vital molecules expressed in ROS-mediated downstream signaling pathway were tested by Western blotting. As shown in Fig. 9A, compared with SeNPs and curcumin, the expression of p53 was in a downregulated trend after treatment with Se@Cur. Similarly, for the AKT signaling pathway, Se@Cur markedly promoted the protein expression of AKT (Fig. 9B). In general, the present results indicated that Se@Cur induced HepG2 cells apoptosis by at least two ROS-mediated signaling pathways.

\subsection{In vivo anticancer activity of Se@Cur}

In vivo anticancer effect of Se@Cur is an important indicator for the application in the medical field. ${ }^{49}$ Therefore, the dose dependent experiment of tumor weight and body weight were performed to evaluate its in vivo anticancer activity. As shown in Fig. 10A, the HepG2 xenogragts nude mice were treated with Se@Cur at the dose of $3,6,9 \mathrm{mg} \mathrm{kg}^{-1}$ for a period of 16 days, and the tumor weight after last treatment were $0.81 \pm 0.03 \mathrm{~g}$, $0.60 \pm 0.04 \mathrm{~g}$ and $0.31 \pm 0.02 \mathrm{~g}$, respectively. Similarly, after treatment with different concentration of Se@Cur, the body weights were $16 \pm 0.46 \mathrm{~g}, 15 \pm 0.41 \mathrm{~g}$ and $14 \pm 0.42 \mathrm{~g}$, respectively (Fig. 10B). Taken together, compare to control group, there was no statistically significant difference in body weight, but no distinct reduction was observed in the body weight. Images of the mice with different size of tumors were showed in Fig. S4. $\dagger$ The dates suggested that Se@Cur significantly inhibited the growth of tumor while exhibited low side effect. Taken together, these results indicated the effective in vivo anticancer capacity of Se@Cur.

\section{Conclusions}

In conclusion, this paper demonstrated a chemical method to synthesize curcumin loaded selenium nanoparticle (Se@Cur). Se@Cur exhibited superior abilities to enhance the solubility of drug, inhibit cell proliferation and suppress cell migration. Furthermore, the mechanistic investigation revealed that Se@Cur activated caspase-3 mediated HepG2 cells apoptosis by ROS overproduction. Moreover, Se@Cur induced HepG2 cells apoptosis via P53 and AKT signaling pathways. The in vivo experiments showed injection of mice with Se@Cur effectively 
inhibited the tumor growth. Taken together, for achieving anticancer activity, the strategy to use SeNPs as a carrier of curcumin could be a highly efficient way. Se@Cur may be a candidate for next assessment as a chemotherapeutics of cancers, especially HCC.

\section{Conflicts of interest}

There are no conflicts to declare.

\section{Acknowledgements}

This work was supported by the Technology Planning Project of Guangzhou (2018-1002-SF-0673), the Technology Planning Project of Guangdong Province (No. 2014A020212697), the Technology Planning Project of Guangdong (No. 201607010120), The Science and Technology Planning Project of Guangdong Province (No. 2014A020212024),The Science and Technology Planning Project of Guangdong Province (No. 2015A020211002), the Medical science and technology project of Guangzhou Municipal Health Bureau (No. 0005559A11105033).

\section{Notes and references}

1 J. Lan, N. Wang, L. Huang, Y. Liu, X. Ma, H. Lou, C. Chen, Y. Feng and W. Pan, Eur. J. Med. Chem., 2017, 127, 554-566. 2 B. Zhu, L. Sun, W. Luo, M. Li, D. H. Coy, L. Yu and W. Yu, Oncotarget, 2017, 8, 23289-23302.

3 M. Li, W. Zhang, B. Wang, Y. Gao, Z. Song and Q. C. Zheng, Int. J. Nanomed., 2016, 11, 5645-5669.

4 A. G. Duffy, S. V. Ulahannan, O. Makorova-Rusher, O. Rahma, H. Wedemeyer, D. Pratt, J. L. Davis, M. S. Hughes, T. Heller, M. ElGindi, A. Uppala, F. Korangy, D. E. Kleiner, W. D. Figg, D. Venzon, S. M. Steinberg, A. M. Venkatesan, V. Krishnasamy, N. Abi-Jaoudeh, E. Levy, B. J. Wood and T. F. Greten, J. Hepatol., 2017, 66, 545-551.

5 Z. H. Ye, L. Gao, D. Y. Wen, Y. He, Y. Y. Pang and G. Chen, OncoTargets Ther., 2017, 10, 1711-1723.

6 X. F. Zhang, X. Yang, H. L. Jia, W. W. Zhu, L. Lu, W. Shi, H. Zhang, J. H. Chen, Y. F. Tao, Z. X. Wang, J. Yang, L. X. Wang, M. Lu, Y. Zheng, J. Zhao, Q. Z. Dong and L. X. Qin, Cancer Biol. Med., 2016, 13, 459-468.

7 R. Lencioni, J. M. Llovet, G. Han, W. Y. Tak, J. Yang, A. Guglielmi, S. W. Paik, M. Reig, D. Y. Kim, G. Y. Chau, A. Luca, L. R. del Arbol, M. A. Leberre, W. Niu, K. Nicholson, G. Meinhardt and J. Bruix, J. Hepatol., 2016, 64, 1090-1098.

8 R. Dutta and R. I. Mahato, Pharmacol. Ther., 2017, 173, 106117.

9 J. H. Yoon, J. I. Choi, Y. Y. Jeong, A. Schenk, L. Chen, H. Laue, S. Y. Kim and J. M. Lee, J. Hepatol., 2016, 65, 1155-1162.

10 G. Roviello, L. Zanotti, M. R. Cappelletti, A. Gobbi, G. Borsella, C. Pacifico, A. G. Multari and D. Generali, Crit. Rev. Oncol. Hematol., 2016, 108, 62-68.

11 C. H. Wang, K. C. Wey, L. R. Mo, K. K. Chang, R. C. Lin and J. J. Kuo, Asian Pac. J. Cancer Prev., 2015, 16, 3595-3604.
12 R. Barbara, D. Belletti, F. Pederzoli, M. Masoni, J. Keller, A. Ballestrazzi, M. A. Vandelli, G. Tosi and A. M. Grabrucker, Int. J. Pharm., 2017, 526, 413-424.

13 M. A. Abdel-Wahhab, A. S. Salman, M. I. Ibrahim, A. A. ElKady, S. H. Abdel-Aziem, N. S. Hassan and A. I. Waly, Food Chem. Toxicol., 2016, 94, 159-171.

14 W. R. Garcia-Nino and J. Pedraza-Chaverri, Food Chem. Toxicol., 2014, 69, 182-201.

15 S. Kim, M. J. Stebe, J. L. Blin and A. Pasc, J. Mater. Chem. B, 2014, 2, 7910-7917.

16 W. Zhong, K. Qian, J. Xiong, K. Ma, A. Wang and Y. Zou, Biomed. Pharmacother., 2016, 83, 302-313.

17 L. Wang, X. Chen, Z. Du, G. Li, M. Chen, X. Chen, G. Liang and T. Chen, J. Exp. Clin. Cancer Res., 2017, 36, 47.

18 M. Kumari, L. Ray, M. P. Purohit, S. Patnaik, A. B. Pant, Y. Shukla, P. Kumar and K. C. Gupta, Eur. J. Pharm. Biopharm., 2017, 117, 346-362.

19 K. Margulis, S. Srinivasan, M. J. Ware, H. D. Summers, B. Godin and S. Magdassi, J. Mater. Chem. B, 2014, 2, 3745-3752.

20 K. V. Jardim, G. A. Joanitti, R. B. Azevedo and A. L. Parize, Mater. Sci. Eng., C, 2015, 56, 294-304.

21 J. S. Baek and C. W. Cho, Eur. J. Pharm. Biopharm., 2017, 117, 132-140.

22 M. M. Wen, N. S. El-Salamouni, W. M. El-Refaie, H. A. Hazzah, M. M. Ali, G. Tosi, R. M. Farid, M. J. BlancoPrieto, N. Billa and A. S. Hanafy, J. Controlled Release, 2017, 245, 95-107.

23 D. Sobot, S. Mura and P. Couvreur, J. Mater. Chem. B, 2016, 4, 5078-5100.

24 Y. F. Song, Y. Q. Xie, J. J. Yang, R. Q. Li, X. Jin and J. Yang, J. Mater. Chem. B, 2016, 4, 6588-6596.

25 L. B. Naves, C. Dhand, J. R. Venugopal, L. Rajamani, S. Ramakrishna and L. Almeida, Prog. Biomater., 2017, 6, 13-26.

26 Z. Cui, D. Liu, C. Liu and G. Liu, Medicine, 2017, 96, e5944. 27 L. Wang, J. Wang, X. Liu, Q. Liu, G. Zhang and L. Liang, Biosci. Rep., 2016, 36, e000395.

28 M. Vinceti, G. Dennert, C. M. Crespi, M. Zwahlen, M. Brinkman, M. P. Zeegers, M. Horneber, R. D'Amico and C. Del Giovane, Cochrane Database Syst. Rev., 2014, 3, CD005195.

29 Y. Zhang, X. Li, Z. Huang, W. Zheng, C. Fan and T. Chen, Nanomedicine, 2013, 9, 74-84.

30 P. D. Terry, B. Qin, F. Camacho, P. G. Moorman, A. J. Alberg, J. S. Barnholtz-Sloan, M. Bondy, M. L. Cote, E. Funkhouser, K. A. Guertin, E. S. Peters, A. G. Schwartz, J. M. Schildkraut and E. V. Bandera, J. Nutr., 2017, 147, 621-627.

31 X. Cai, C. Wang, W. Yu, W. Fan, S. Wang, N. Shen, P. Wu, X. Li and F. Wang, Sci. Rep., 2016, 6, 19213.

32 D. L. Hatfield, P. A. Tsuji, B. A. Carlson and V. N. Gladyshev, Trends Biochem. Sci., 2014, 39, 112-120.

33 F. Maiyo and M. Singh, Nanomedicine, 2017, 12, 1075-1089. 34 M. Zuberek, D. Wojciechowska, D. Krzyzanowski, S. Meczynska-Wielgosz, M. Kruszewski and A. Grzelak, J. Nanobiotechnol., 2015, 13, 72. 
35 H. Zhang, A. M. Gomez, X. Wang, Y. Yan, M. Zheng and H. Cheng, Cardiovasc. Res., 2013, 98, 248-258.

36 L. A. Sena and N. S. Chandel, Mol. Cell, 2012, 48, 158-167. 37 J. Zhang, X. Wang, V. Vikash, Q. Ye, D. Wu, Y. Liu and W. Dong, Oxid. Med. Cell. Longevity, 2016, 2016, 4350965.

38 Y. Li, Z. Lin, M. Zhao, T. Xu, C. Wang, H. Xia, H. Wang and B. Zhu, Int. J. Nanomed., 2016, 11, 3065-3076.

39 Y. H. Li, Z. F. Lin, T. T. Xu, C. B. Wang, M. Q. Zhao, M. S. Xiao, H. Z. Wang, N. Deng and B. Zhu, RSC Adv., 2017, 7, 1453-1463.

40 Y. H. Li, Z. F. Lin, M. Q. Zhao, T. T. Xu, C. B. Wang, L. Hua, H. Z. Wang, H. M. Xia and B. Zhu, ACS Appl. Mater. Interfaces, 2016, 8, 24385-24393.

41 Y. H. Li, Z. F. Lin, M. Q. Zhao, M. Guo, T. T. Xu, C. B. Wang, H. M. Xia and B. Zhu, RSC Adv., 2016, 6, 89679-89686.

42 Y. Li, M. Guo, Z. Lin, M. Zhao, M. Xiao, C. Wang, T. Xu, T. Chen and B. Zhu, Int. J. Nanomed., 2016, 11, 6693-6702.
43 B. Zhu, Y. Li, Z. Lin, M. Zhao, T. Xu, C. Wang and N. Deng, Nanoscale Res. Lett., 2016, 11, 198.

44 Y. H. Li, X. L. Li, Y. S. Wong, T. F. Chen, H. B. Zhang, C. R. Liu and W. J. Zheng, Biomaterials, 2011, 32, 9068-9076.

45 Z. F. Lin, Y. H. Li, M. Guo, T. T. Xu, C. B. Wang, M. Q. Zhao, H. Z. Wang, T. F. Chen and B. Zhu, RSC Adv., 2017, 7, 742750.

46 Y. H. Li, X. L. Li, W. J. Zheng, C. D. Fan, Y. B. Zhang and T. F. Chen, J. Mater. Chem. B, 2013, 1, 6365-6372.

47 N. M. Khan, A. Haseeb, M. Y. Ansari, P. Devarapalli, S. Haynie and T. M. Haqqi, Free Radical Biol. Med., 2017, 106, 288-301.

48 G. Wang, T. Zhang, W. Sun, H. Wang, F. Yin, Z. Wang, D. Zuo, M. Sun, Z. Zhou, B. Lin, J. Xu, Y. Hua, H. Li and Z. Cai, Free Radical Biol. Med., 2017, 106, 24-37.

49 Y. Huang, L. He, W. Liu, C. Fan, W. Zheng, Y. S. Wong and T. Chen, Biomaterials, 2013, 34, 7106-7116. 